\title{
DESIGN AND DEMONSTRATION OF A RADIOISOJET
}

\section{R. VIVENTI, JR. W. C. ISLEY}
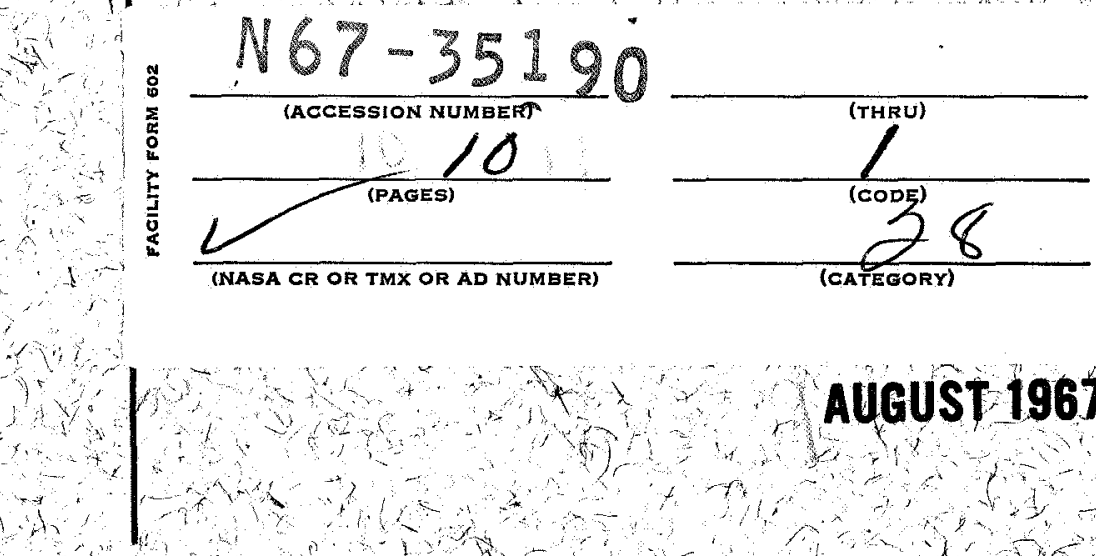

NASA

\section{GODDARD SPACE FLIGHT CENTER GREENBELT, MARYLAND}

Paper prepared for presentation to ÂlAA 3rd Propulsion Joint Specialist Conference, Washingtion, D. C.-July 17-21, 1967 


\title{
DESIGN AND DEMONSTRATION
}

OF A RADIOISOJET

\author{
R. Viventi, Jr. \\ General Electric Company \\ Cincinnati, Ohio \\ W. C. Isley \\ NASA-Goddard Space Flight Center \\ Greenbelt, Maryland
}

\begin{abstract}
AUGUST 1967
GODDARD SPACE FLIGHT CENTER

Greenbelt, Maryland
\end{abstract}

Paper prepared for presentation to AIAA 3rd Propulsion Joint Specialist Conference, Washington, D. C. - July 17-21, 1967 


\author{
R. Viventi, Jr. \\ General Electric Company \\ Cincinnati, Ohio \\ W. C. Isley \\ NASA Goddard Space Flight Center \\ Greenbelt, Maryland
}

\section{Abstract}

A joint NASA/AEC effort was undertaken to develop and evaluate the performance of a radioisotope heated thruster for possible spacecraft application. A Pm-147 oxide capsule was used as the source of heat in a nominal 20 millipound ammonia thruster. The mechanical design of the thruster was based on that of the thermal storage resistance jet. Measurements were made of thrust, propellant mass flow, and temperature transients during a continuous two-week test program. Correlation with an electrically heated unit was excellent. The nominal 60 watt (thermal) source produced core temperatures in excess of $1650^{\circ} \mathrm{F}$ and resulted in measured values of specific impulse greater than $230 \mathrm{sec}$ at 22 millipounds thrust. Thruster performance parameters were measured over the full range of duty cycle and as a function of propellant flow rate. The results were found to correlate with duty cycle. Application of the radioisojet to a number of missions has been considered. Long life, low thrust applications, for which the power penalty must be borne by the auxiliary propulsion system, are attractive in terms of weight, reliability, and cost.

\section{Introduction}

The "Radioisojet" Thruster concept inherently involves the characteristics of a thermal storage device where heat is generated at a fixed rate by radioisotope decay within an internal capsule. Through efficient shielding, propelilants can be heated to temperatures sufficiently high to permit a factor of from 2 to 3 improvement in specific impulse over that obtainable as a cold gas. Such a thruster can be designed from either of two approaches.

The first approach is to build a pulse mode type of thruster which utilizes energy stored during propellant off periods to raise the temperature of the internal core to the highest possible point, whereupon propellant is pulsed through the unit for brief periods during operation. This avenue of design naturally places considerable importance on thruster duty cycle and pulse duration in order to achieve highest possible specific impulse. As pulse duration increases, the average specific impulse obtainable decreases. Using this method, improvements in performance axe possible only by increasing the thermal storage capability.

The second design approach is to size the thermal source for a given thrust level and specific impulse under continuous flow. This avenue presents the design problem of thermal control (heat rejection) during the no-flow periods or a physical design which permits temperature cycling above the full-flow value.
The selection of best design approach is closely tied to the particular mission requirement. For example, a device required for low thrust prime spacecraft propulsion is generally representative of long-term or essentially continuous thrust application over a short trajectory segment. For this condition, it can be demonstrated analytically that best performance is achieved when the second (constant thrust) design approach is employed. For spacecraft orbit trim, station keeping, and attitude control functions, the typical mission requirement is usually best suited to a pulse mode type of thruster.

The design approach is also quite dependent upon the type of radioisotope source to be employed. Sources of low power density may not be capable of performing competitively in a constant thrust mode because of heat shielding limitations and heat transfer problems. Radioisotope half life is also very important, as related to the mission requirement.

A thruster required for 2 to 3 year station keeping operation may have to consider heat rejection at the beginning of the mission to maintain a more constant power level due to half-life of the source.

\section{Principles of Operation}

The radioisojet is an advanced thruster design which utilizes the thermal energy generated during the radioactive decay of a radioisotope--in this case, promethium 147--to heat a propellant to operating temperature. This concept is a logical extension of the existing resistance jet technology wherein the propellant is heated by the thermal energy generated in a resistance wire. As shown in the exploded view of Figure 1 , the radioisojet consists of three major subassemblies: the heat shielding package, the thruster body, and the radioisotope fueled heater. Comparable resistance jet components having similar functions are shown in Figure 2. The assembly of the isotope-fueled heater into the thruster body provides a narrow flow annulus $(0.020$ in.) for the gaseous ammonia. This annulus permits metal-to-gas heat transfer to take place, raising the ammonia temperature sufficiently high to cause decomposition into hydrogen and nitrogen. Decomposition is desirable because of the increase in specific impulse which can be obtained in comparison with molecular ammonia for a given temperature.

Propellant flow control is provided by a low power axial solenoid valve supported from the thruster at the mounting flange. The time required for travel of the valve poppet from fully closed to fully open is less than 100 milliseconds, thereby allowing the thruster to be operated in a small impulse bit mode as well as in steady state. 
The radioisojet thruster is designed for operation in a pulsed mode where maximum core temperature is low enough to eliminate a requirement for cooling during no-flow periods. The requixed isotopic power for a given temperature is minimized through the use of a multilayer heat shielding package consisting of concentric cylinders of thin Nichrome $V$ foil separated by a minimum number of coils of small diameter Nichrome $v$ wire. All radiating surfaces of the thruster body except for the exhaust nozzle and the propellant inlet tube are surrounded by 40 layers of this heat shielding.

The original design analysis conducted to determine heat shielding effectiveness indicated that with 40 layers of the above heat shielding, a body temperature of $2000^{\circ} \mathrm{F}$ could be reached with a 60 watt input, as shown in Figure 3 . Subsequent design modifications required for support of the capsule heater necessitated a corresponding change in the math model to account for increased radiation from a higher temperature in the mounting flange area. The modified design model predicted performance is also shown in Figure 3 . This comparison indicates a reduction in operating temperature to approximately $1900^{\circ} \mathrm{F}$ at 60 watts input. The modified design was used as a basis for the construction of actual thruster hardware.

\section{Design Considerations}

Studies were performed prior to initiation of actual thruster design to determine the most suitable radioisotope heat source in terms of acceptable power density, handling and proximity hazards, half life, and availability for this program. Radioisotopes having half lives less than 2 years were discarded on the basis of the projected mission applications for this type of thruster. The beta sources were considered more desirable from the engineering standpoint because they circumvent the problem of containment or venting of evolved helium, as is necessary with alpha sources such as plutonium 238. Promethium 147 oxide was found to be the best overall compromise in terms of the above factors and from the standpoint of availability for this program.

Some selected properties of this radioisotope $\left(\mathrm{Pm}_{2} \mathrm{O}_{3}\right)$ are summarized below:

$\begin{array}{ll}\text { Half life } & 2.620 \pm 0.005 \mathrm{yr} \\ \text { Power density } & 2.09 \text { watts/cc } \\ \text { Melting point } & 2270^{\circ} \mathrm{C} \\ \text { Density } & 7.3 \mathrm{grams} / \mathrm{cc} \\ \text { Pm } 147 \text { content } & 86 \% \mathrm{by} \text { weight } \\ \text { Specific activity of } & 928.4 \pm 1.7 \mathrm{curies} / \mathrm{gm}\end{array}$

Pm 147 metal

A considerable savings in cost and time expenditures was realized during chemical compatibility screening tests by using samarium oxide to find the most promising cladding material for use in the final capsule configuration. Since samarium oxide is not radioactive, all such handling hazards were avoided during these tests.

The radioisojet design approach separates the promethium heat source from the propeliant by

\footnotetext{
* Promethium Isotopic Power Data Sheets - BNWC-45Battelle Northwest
}

encapsulating the clad source in a Hastelloy $\mathrm{X}$ * inner body. The outer surface of the Hastelloy $x$ heater slug provides an inner annular surface of the propellant flow path.

Hastelloy $X$ was chosen for the annular surface material as a result of earlier electrically heated thruster tests where it was found that with this material in contact with the ammonia, catalytic decomposition was promoted at temperatures as low as $1500^{\circ} \mathrm{F}$.

An important design feature built into the radioisojet thruster is the ability to obtain performance data with an electrical simulator mounted in place of the promethium 147 heater. The electrically heated inner body, built around a simple platinum-rhodium wire wound resistance heater, is inserted into the outer body and heat shielding subassembly for the preliminary tests and then removed and replaced by the radioisotope heater for final performance tests. With the modular three unit assembly as shown in Figure 1, either the heater or the heater/thruster subassembly can be removed without disturbing the heat shielding package.

The selected nominal operating thrust level of the radioisojet thruster was 20 millipounds at a pressure of 1.5 atmospheres, using ammonia propellant. The nozzle divergent section contour is based upon proven thermodynamic and fluid dynamic analyses which have been computerized to provide rapid solutions to iterative processes. Flow is treated by a hypothesis wherein it is assumed that thermodynamic equilibrium is maintained up to the Mach 1 condition and composition is frozen thereafter. The fluid dynamic analysis defines optimum nozzle contour within the confines of acceptable length and nozzle exit diameter. Boundary layer growth in the divergent section of the nozzle and thrust efficiency are also defined as a function of operating temperatures and inlet pressures. A tungsten probe has been used in some earlier tests with a nozzle of comparable performance to verify the velocity profile analysis which shows that approximately $40 \%$ of the exit diameter is taken up by the boundary layer as predicted.

Total radioisojet system weight is one important consideration since it forms a basis for direct comparison to a resistance jet of similar performance. With the electrical heater in place of the promethium-fueled capsule, the radioisojet was found to weigh almost exactly 5 1b. With the isotope in place, the weight of the system is estimated to be the same within $0.251 \mathrm{~b}$.

A similar state-of-the-art resistance jet currently weighs 1.7 lb but requires an additional $5 \mathrm{lb}$ of power supply weight (assuming 7 watts/1b from a solar panel array and a 35 watt power requi rement).

As the distance from the sun increases, the resistance jet starts paying a higher weight penalty due to reduced solar panel output, while the radioisojet weight stays constant.

A preliminary investigation of re-entry of a radioisojet has been made although no specific design features to provide for it have been incorporated into the present configuration. Both intact re-entry and high altitude dispersion can 
be considered, the latter being potentially possible due to the small amount of isotope being carried in a thruster. If intact re-entry appears to be the more attractive approach, the existing design would have to be modified to provide the necessary capsule protection.

\section{Experimenta1 Results}

A benchmark power-temperature measurement was made in the General Electric Propulsion Laboratory with the electrical heater installed prior to initiation of the "hot" fueled testing. The primary temperature measurement was made at the center of the heater element in the hottest portion of the heater. At the 60 watt power input level, the measured temperature in the heater core was $1850^{\circ} \mathrm{F}$. The steady state temperatures reached over a range of power inputs from 13.5 to 65 watts during this preliminary testing are shown in curve A of Figure 4. Subsequently, the thruster in the same configuration was installed in the test facility at Mourtd Laboratories and the temperature/power relationship checked in the same manner. These results appear as curve $B$ of Figure 4 . The same relationship was rechecked following the radioisojet performance tests while still installed in the Mound Facility and curve $C$ of Figure 4 generated. The final check of power/temperature (curve D) was made at the General Electric Propulsion Laboratory.

The difference in temperature measured at the General Electric and Mound facilities at a constant power input of 60 watts can be explained only by a variation in the effectiveness of the heat shielding caused by surface oxidation, since no parameters were changed between tests except for an increase in the ambient pressure level from $10^{-7}$ torr in the General Electric facility to $10^{-3}$ torr in the Mound facility chamber.

Conductive heat transfer between adjacent layers of heat shielding might be increased but not significantly. Since a change in heat shielding emissivity from .3 to .4 could account for the difference between $1650^{\circ} \mathrm{F}$ and $1850^{\circ} \mathrm{F}$ at 60 watts power level, it is conceivable that the increased oxidation resulting from the poorer vacuum was the cause. This is further evidenced by the shift in curve $D$ towards the original data curve after being reinstalled in the GE propulsion lab facility where, under improved vacuum, surface oxides were volatilized, thus reducing heat shield emissivity.

Thrust measurements were made with the radioisojet mounted on a displacement type balance supported by three thin flexure straps. Two identical balances were fabricated with one installed at the GE facility and the other installed at the Mound facility. This latter installation with the radioisojet mounted in place is shown in Figure 5. The sensitivity of this thrust device is such that a force of one millipound is sufficient to produce a lateral displacement of $0.75 \mathrm{mils}--1 \mathrm{arge}$ enough to be readily sensed by a linear variable displacement transducer. During the preliminary tests, thrust levels were measured between 5 and 40 millipounds. A calibrated orifice, located just upstream of the thruster inlet tube, was used to determine propellant flow rate from which an instantaneous specific impulse was calculated from the relationship: Specific impulse = thrust/propellant weight flow rate.
Upon completion of preliminary tests at GE, the electrically heated thruster was transferred to the test facility at Mound Laboratories and satisfactory operation of the facility was confirmed by comparing performances obtained in identical thrust balances at the two separate locations.

The electrically heated thruster was removed from the heat shielding package and the radioisotope fueled thruster installed while mounted in the balance. "Hot" testing was initiated shortly thereafter with a series of thrust measurements obtained during relatively short ( 5 to 10 seconds) pulses over a range of chamber pressures from 5 psia to 25 psia. As with the electrical tests, specific impulse calculations were made based on the propellant flow rate through a calibrated orifice. The resulting curve of specific impulse vs chamber pressure of Figure 6 took a form typical of that obtained with electrically heated thrusters. The maximum calculated specific impulse of 232 seconds was derived from thrust data obtained near the design chamber pressure of 1.5 atmospheres with the slope of the curve diminishing to zero at that pressure, indicating that a maximum specific impulse level was reached as predicted.

Since the promethium fuel had been completely encapsulated in a refractory liner and then welded into a Hastelloy X slug, closed at both ends, no direct core temperature measurement could be made during the radioisotope fueled thruster testing. The closest available location for a thermocouple adjacent to the hot core was in a hole bored into the rear dome which placed its junction approximate1y $1 / 16$ in. from the outside surface of the capsule cladding. The temperature measured here was $1450^{\circ} \mathrm{F}$ at the onset of the testing. A correlation of outer surface temperatures for the electrical and promethium fueled units indicated a core temperature of $1800^{\circ} \mathrm{F}$ for the isotope fueled thruster.

A lengthy test series was conducted operating the thruster in the pulsed mode, monitoring heater temperature until steady state equilibrium conditions were reached. Duty cycles from $0.5 \%$ to $100 \%$ were investigated at three thruster chamber pressure levels--1,1.5, and 2 atmospheres. During these tests, steady state was defined as occurring at the time when no greater than a 5 degree per hour change in temperature was measured for a three-hour period. A typical curve obtained during the testing at 1 atmosphere is shown in Figure 7 where, with a 30 second on, 100 second off cycle, steady state was not reached until almost $\mathbf{3 5 0}$ minutes had elapsed.

In all, 45 steady state measurements were made. After converting the ratio of on/off time to a duty cycle, the summary curve of Figure 8 was plotted. In this plot, the effect of minimum temperature reached was found to be independent of pulse duration and dependent only upon the ratio of time on/time off. In order to determine maximum temperature decay at each pressure level, the thruster was allowed to reach a minimum temperature with the valve fully open. The base temperature reached at each of the three pressures is plotted at $100^{\circ}$ duty cycle.

Since thrust and propellant flow rate were continuously monitored during these tests, the base temperature reached could be converted to a specific impulse. Figure 9 illustrates the data obtained at 
1 atmosphere to show a drop in specific impulse from a maximum of over 230 seconds to 150 seconds at full flow, corresponding to a drop in core temperature to $800^{\circ} \mathrm{F}$ (from Figure 7 ).

At one point during this testing, the vacuum chamber was flooded with nitrogen gas in an attempt to simulate the heat transfer conditions which could exist between adjacent layers of heat shielding if the thruster were mounted on a spacecraft awaiting launch at a sea level launch pad. The resulting equilibrium temperature of $965^{\circ} \mathrm{F}$ may be sufficiently low to permit normal system integration with the spacecraft without the necessity for cooling or oxidation protection.

Subsequent to the above testing, the radioisojet was removed from the Mound facility, the thruster outer body separated from the heater, and an electrical heater inserted. Additional tests were performed at the GE facility to determine further correlation with the two modes of heating. Tests were performed at room temperature, $1950^{\circ} \mathrm{F}, 1800^{\circ} \mathrm{F}$, $1650^{\circ} \mathrm{F}$, and $1550^{\circ} \mathrm{F}$. The resulting specific impulse values obtained are plotted in Figure 10.

The measured value of 232 seconds obtained with the promethium-fueled thruster at 25 psia corresponds almost exactly with the $1660^{\circ} \mathrm{F}$ electrically heated thruster data obtained at the same thruster chamber pressuxe. From this correlation, it can thus be concluded that the heater core temperature during the promethium-fueled thruster tests was approximately $1650^{\circ} \mathrm{F}$.

The thruster has since been reassembled with the radioisotope heater and currently is mounted in a vacuum facility at Battelle Northwest Laboratories. Long-term tests are being conducted to confirm capsule/cladding compatibility and influence of ammonia propellant on thruster materials.

\section{Mission Applications}

There are a number of potential applications for a radioisojet propulsion system. These range from long-term earth orbital missions devoted to communications, meteorology, and earth resources up through deep space probes or other planetary orbiters. From a mission standpoint, the radioisojet has the advantage that it requires no electrical power from the spacecraft for heating of propellant and is therefore functionally independent from other spacecraft systems. A second major factor is the inherently high reliability of such a device for long-term operation because of the predictable nature of the radioisotope decay mechanism and the elimination of electrical heaters and associated power conditioning.

An attractive application would be the use of such a system in the form of multijet modules (each consisting of four jets having a common heater) for orbit adjustment and attitude stabilization of advanced 24-hour symchronous spacecraft. Such a system can be designed to permit sizing of attitude jets to one particular thrust level while sizing orbit adjustment jets to one particular thrust level required for the acquisition phase. Using pulse mode operation, the orbit adjustment jets can also satisfy the station keeping requirement. Other low thrust devices, such as the ion engine, require either excessive amounts of power or extremely long operational periods to fulfill all of these mission requirements.

It is possible to extrapolate the design of the single jet radioisotope thruster to an advanced multijet version by means of data already compiled on electrically heated multijet thrusters. On this basis, it is estimated that the multijet version would weigh approximately 5.75 pounds, exclusive of any radiation shield required by aerospace safety, for a given mission. Performance calculations were made for a 1,500-pound synchronous spacecraft which has a mission life of three years. The propulsion system would be required to provide east-west and north-south station keeping as well as attitude stabilization on three axes. During initial acquisition, the propulsion system would have to remove injection errors and maneuver the spacecraft to the proper longitude. The radioisojet system required for this application was sized using performance data measured in this test program. For a total impulse requirement of 12,500 poundseconds, the ammonia propellant inventory was found to be 57 pounds at an average mission specific impulse of 220 seconds.

Total system weight, including four modules (exclusive of re-entry shielding), propellant, storage tank, and feed system components, was estimated to be 100 pounds. A resistance jet system designed to perform this mission would weigh approximately the same, since a thruster weight savings would be balanced by the weight of the requixed power supply. An additional weight savings is available with the radioisojet, since no propellant contingency is required to compensate for specific impulse reduction resulting from potential heater failure. For such applications as deep space probes, the reduced solar panel output or the high battery weight requirements further penalize the electrothermal system in favor of the radioisojet.

In any case, the radioisojet, due to its inherent design simplicity, offers a major improvement in overall spacecraft secondary propulsion reliability.

Adaptation of the radioisojet thruster to specific mission requirements in terms of spacecraft integration and aerospace safety constitutes the next major step toward flight application. 


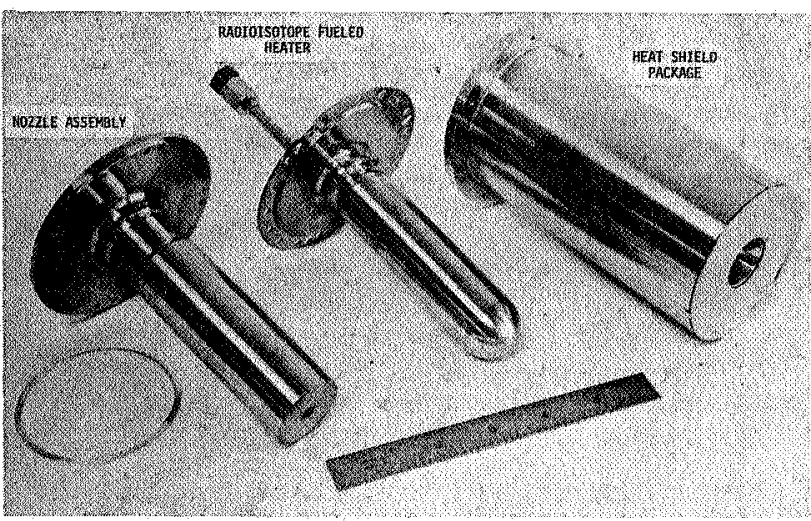

FIGURE 1. EXPLODED VIEW OF RADIOISOJET.

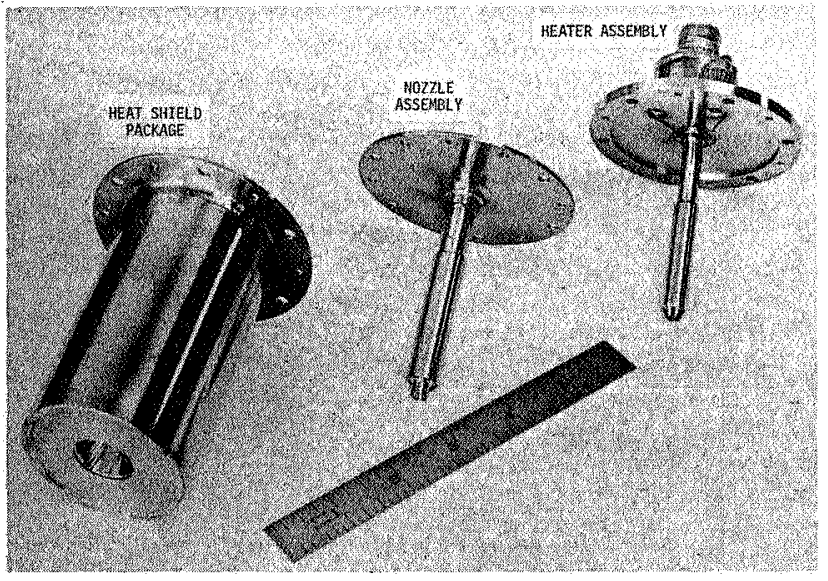

FIGURE 2. EXPLODED VIEW OF RESISTANCE JET.

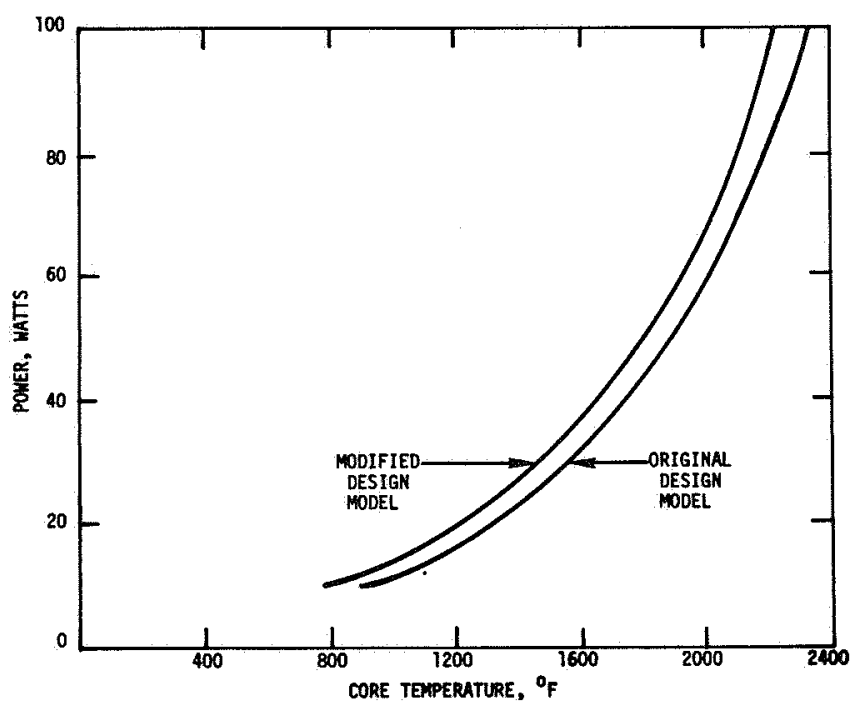

FIGURE 3. RADIOISOJET DESIGN MODEL.

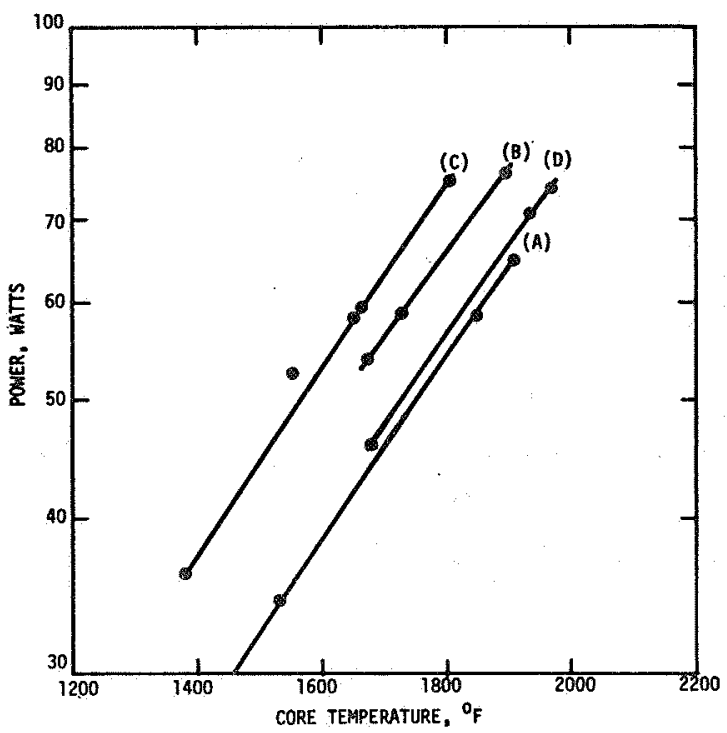

FIGURE 4. ELECTRICAL POWER VERSUS CORE TEMPERATURE.

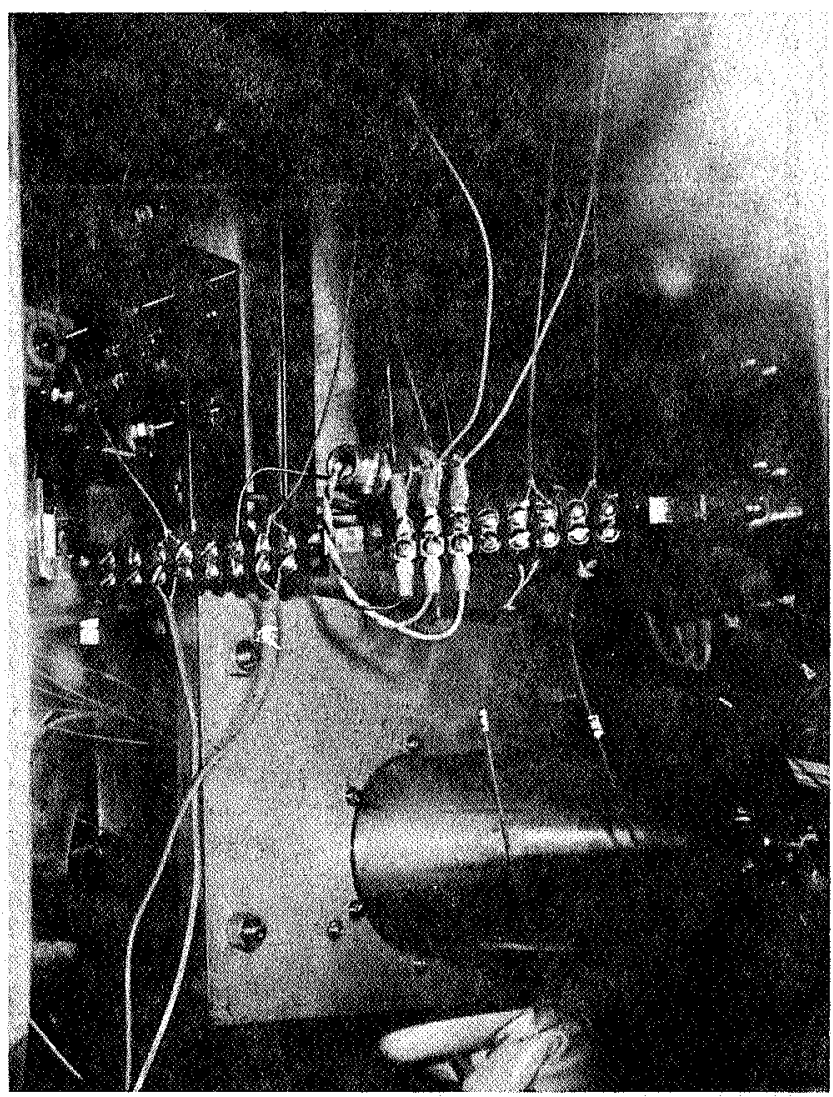

FIGURE 5. MILLIPOUND THRUST RIG. 


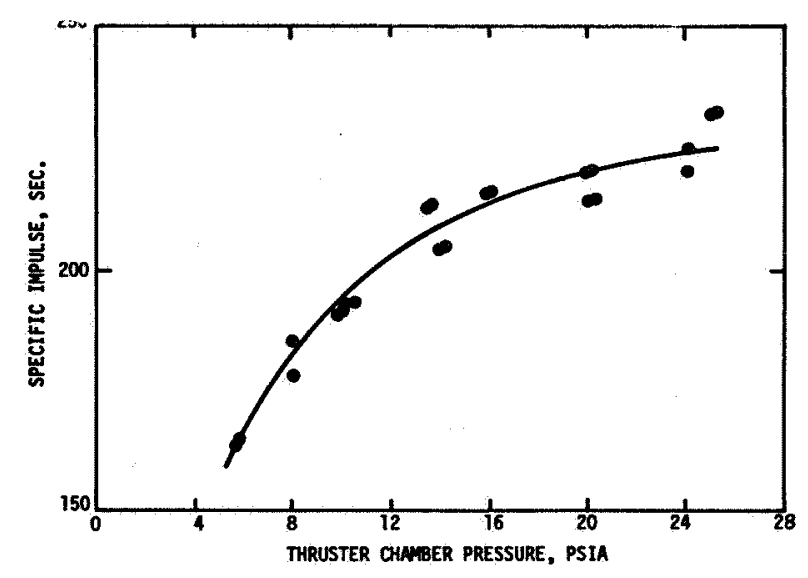

FIGURE 6. RADIOISOJET THRUSTER SPECIFIC IMPULSE VERSUS CHAMBER PRESSURE.

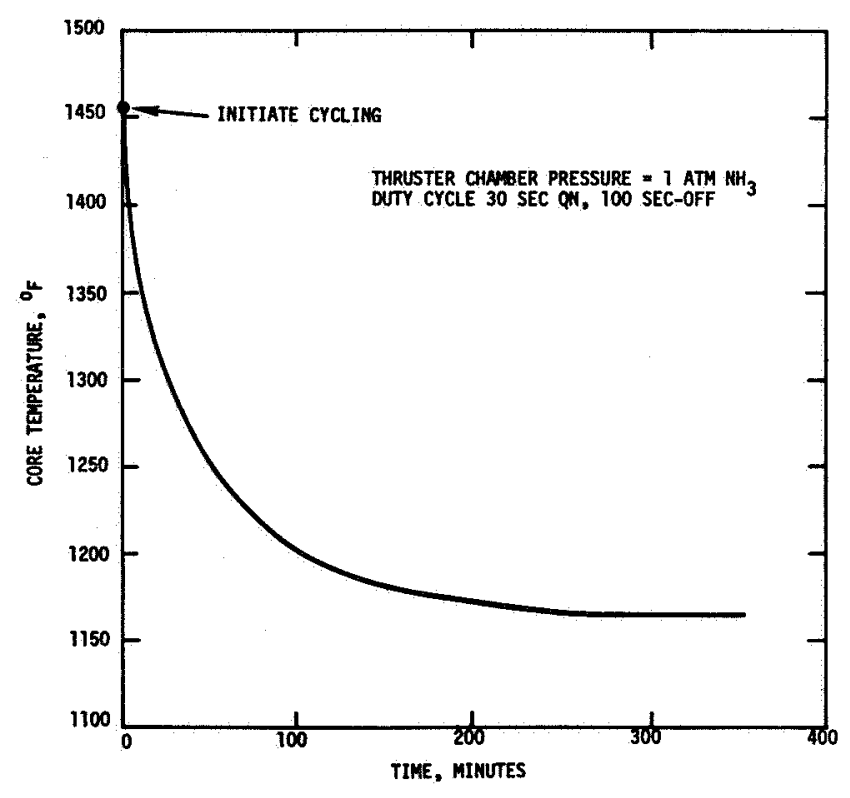

FIGURE 7. RADIOISOJET TEMPERATURE DECAY CURVE.

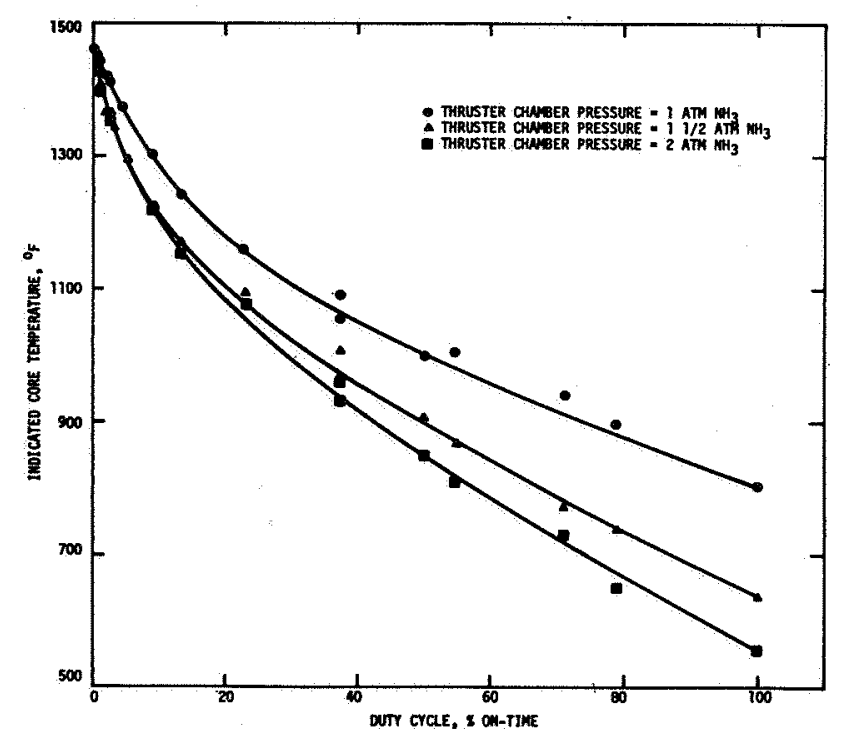

FIGURE 8. STEADY STATE TEMPERATURE VERSUS DUTY CYCLE.

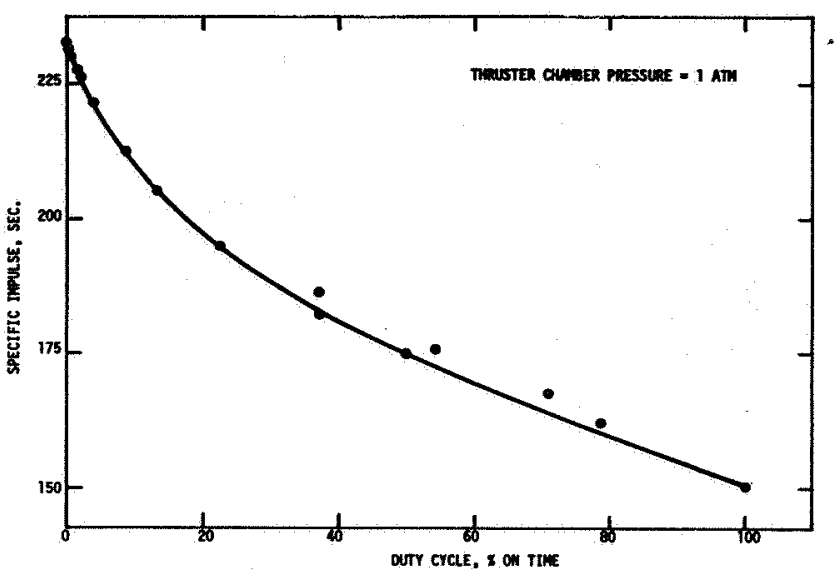

FIGURE 9. RADIOISOJET THRUSTER SPECIFIC IMPULSE VERSUS DUTY CYCLE.

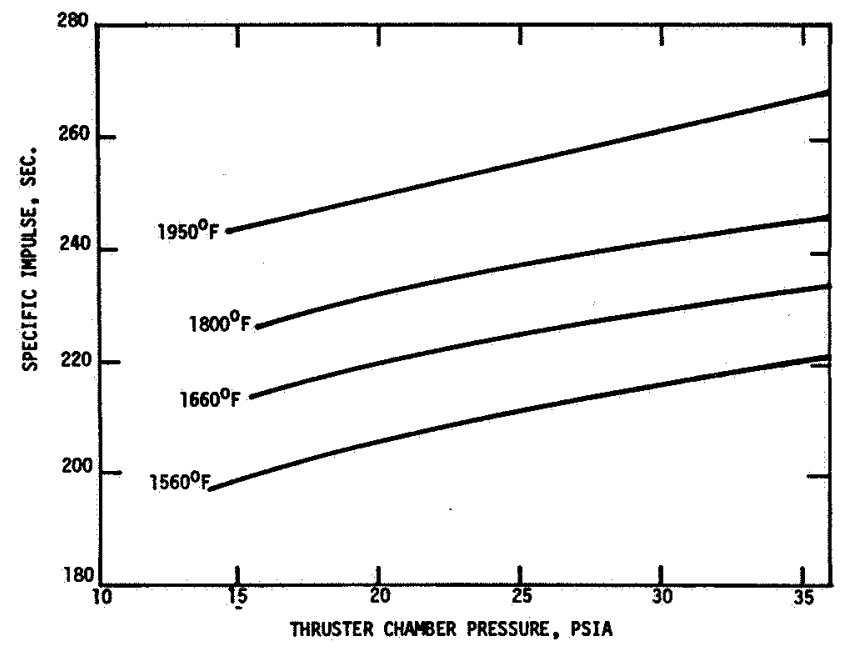

FIGURE 10. ELECTRICALLY HEATED THRUSTER - SPECIFIC IMPULSE VERSUS CHAMBER PRESSURE. 